\title{
Increased Blood Pressure Variability Predicts Poor Short-term Functional Outcome
}

Karen Appiah ${ }^{1}$, Mintu Nath ${ }^{1}$, Will Davison ${ }^{2}$, Sara Mazzucco ${ }^{3}$, John Potter ${ }^{2}$, Peter Rothwell ${ }^{3}$, Tom Robinson ${ }^{1,4}$

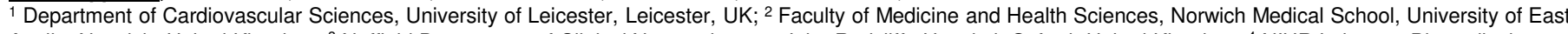
Anglia, Norwich, United Kingdom; ${ }^{3}$ Nuffield Department of Clinical Neurosciences, John Radcliffe Hospital, Oxford, United Kingdom; ${ }^{4}$ NIHR Leicester Biomedical Research Centre, University of Leicester, Leicester, UK

\section{Background}

Hypertension is the most common, yet most treatable stroke risk factor. A transient hypertensive response is present post-acute ischaemic stroke (AIS), and affects approximately $80 \%$ of AIS patients. Though the natural history suggests a spontaneous BP decline over the subsequent day's post-stroke, it has recently emerged that increases in the spontaneous erratic fluctuations of blood pressure (BP), termed blood pressure variability (BPV) is associated with increased vascular risk. BPV is the overall variability of BP over a period of time, and increasing BPV following AIS may be of prognostic significance.

Aim: We investigated the effects of increasing BPV, defined from enhanced casual BP readings, on short-term functional outcome.

\section{Method}

240 AIS and transient ischaemic attack (TIA) patients from three centres were prospectively studied; enhanced casual BP was measured within 48 hours of symptom onset using OMRON 705-IT (Figure 1). Separated by 10 minute intervals between the two sets of three BP readings, supine BP were recorded in the hemiparetic arm. The OMRON 705-IT provided values for systolic $\mathrm{BP}(\mathrm{SBP})$, diastolic BP (DBP), and heart rate (HR); mean arterial pressure (MAP) and pulse pressure (PP) were subsequently derived from the systolic BP and diastolic BP.

BPV was defined as: standard deviation (SD) and coefficient of variation (CoV); functional outcome at one month was assessed as death or disability (modified Rankin scale (mRS) $\geq 3$ ).

Regression analyses performed used variability indices of SBP, DBP, MAP, PP and HR to predict outcome.

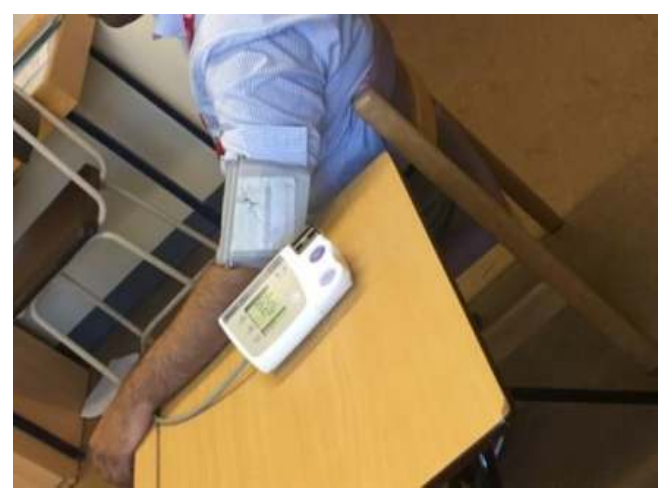

Figure 1

OMRON 705-IT device used to record enhanced casual BP

\section{Results}

Median (IQR) age was 70.5 (64-78); 152 (63.33\%) participants were male, and 219 (91.25\%) were white British. At admission, median (IQR) SBP, DBP and HR were 153mmHg (138-175), $84 \mathrm{mmHg}$ (75-98), and $75 \mathrm{bpm}$ (67-85). Predictors of death and disability at 1 month post-AIS were identified as SD of diastolic $\mathrm{BP}$ and MAP, and CoV of MAP.
These regression models were adjusted for age, sex and baseline $m R S(\geq 3)$. Additionally, other BP parameters, including increasing mean diastolic BP and MAP were also predictors of 1 month functional outcome (Table 1).

\begin{tabular}{|c|c|}
\hline $\begin{array}{l}\text { Predictors of } 1 \text { month } \\
\text { outcome }\end{array}$ & Odds Ratio (95\% Cl), p \\
\hline SBP (mean) & $1.01(1-1.04) ; 0.168$ \\
\hline SBP (SD) & $1.02(0.92-1.13) ; 0.659$ \\
\hline SBP (CoV) & $1(0.86-1.18) ; 0.966$ \\
\hline DBP (mean) & 1.05 (1 to 1.09 ), $0.028^{*}$ \\
\hline DBP(SD) & 1.23 (1.07 to 1.41$), 0.003^{*}$ \\
\hline DBP (CoV) & 1.02 (0.99-1.05); 0.3 \\
\hline MAP (mean) & $1.03(\mathrm{Cl} 1$ to 1.06$), 0.038^{*}$ \\
\hline MAP (SD) & 1.17 (1.03 to 1.34$), 0.018^{*}$ \\
\hline MAP (CoV) & 1.16 (1 to 1.33 ), $0.048^{*}$ \\
\hline PP (mean) & 1 (0.98-1.03); 0.727 \\
\hline PP (SD) & $1.08(0.99-1.18) ; 0.086$ \\
\hline PP (CoV) & 1.05 (0.99-1.1); 0.121 \\
\hline HR (mean) & $1.02(0.98-1.05) ; 0.360$ \\
\hline HR (SD) & $1.11(0.92-1.35) ; 0.273$ \\
\hline HR (CoV) & 1.09 (0.94-1.26); 0.278 \\
\hline
\end{tabular}

Table 1

Increasing BPV, defined using SD and CoV of DBP and MAP were predictors of functional outcome; death and disability were also predicted by mean DBP and MAP

\section{Conclusions}

In this study, we have demonstrated that increasing BPV, particularly in diastolic BP, and MAP, were identified as independent prognostic indicators of the functional outcome at one month post-AIS. 